\title{
Cuestionario sobre el uso de técnicas y manejo de presión del cuff en usuarios adultos con vía aérea artificial por parte de profesionales de salud en Chile
}

\author{
Questionnaire on the techniques and cuff pressure management \\ in artificial airway patients performed by Chilean health \\ professionals
}

Felipe Rosales

Hospital San Juan de Dios
Contacto con el autor:

Felipe Rosales

Santiago - Chile

Correo-e: frosaleslillo@gmail.com

Recibido: $21 / 08 / 2019$

Aceptado: 26/11/2019

\section{RESUMEN}

La evidencia internacional recomienda diferente presión de 'cuff' a utilizar en usuarios con vía aérea artificial. En Chile, algunas instituciones sanitarias han creado protocolos internos referenciando dicha evidencia. Sin embargo, no existe una guía nacional, por lo que distintos profesionales utilizan diferentes criterios. El objetivo de este trabajo fue crear un cuestionario válido y confiable sobre el uso de técnicas y manejo de la presión del 'cuff' en usuarios adultos con vía aérea artificial por parte de profesionales de salud en Chile. Para ello, se utilizó un diseño cualitativo-cuantitativo, descriptivo y longitudinal. El proceso fue dividido en tres fases. En la fase $A$ se desarrolló un cuestionario en inglés que fue traducido al español con ayuda de tres hablantes hispanos nativos. En la fase B diez expertos chilenos en el manejo de usuarios con vía aérea artificial analizaron la herramienta y recomendaron modificaciones de algunos componentes del cuestionario. Después de dichos cambios, la herramienta fue validada a través de un Índice de Validez de Contenido. En la fase $\mathrm{C}$ treinta y tres profesionales de la salud chilenos respondieron voluntariamente el cuestionario en dos ocasiones con un lapso de treinta días entre respuestas, con el fin de medir la confiabilidad a través de 'test-retest'. El cuestionario desarrollado tuvo una validez de 0,93 mientras que $72,73 \%$ y $27,27 \%$ de sus ítems tuvieron excelente y buena confiabilidad respectivamente. Lo anterior permite concluir que este cuestionario cumple con su objetivo y servirá para conocer las técnicas y presiones del 'cuff' que profesionales de la salud en Chile utilizan para el manejo de usuarios adultos con vía aérea artificial.

Palabras clave: vía aérea, traqueostomía, intubación endotraqueal, cuff, cuestionario, Chile.

\begin{abstract}
International evidence recommends different cuff pressure to use with patients with an artificial airway. In Chile, some health institutions have created internal protocols referencing this evidence. However, there is no national guidelines, thus different health professionals use different criteria to manage these patients. The present researched aimed at creating a valid and reliable questionnaire on the use and management of the cuff pressure in adult patients with an artificial airway for health professionals in Chile. A qualitative-quantitative, descriptive and longitudinal design was implemented. This process was divided in three phases. In phase A, a draft of the questionnaire was developed in English and then translated to Spanish by three native Spanish speakers. In phase B, ten Chilean, ten clinical experts in the management of users with artificial airway analysed the tool and recommended modifications of some of the questionnaire. After the modifications, the tool was validated through a Content Validity Index form. In phase C, thirty-three Chilean health professional voluntarily responded the questionnaire twice, with a period of thirty days between responses, to measure the reliability of the tool through 'test-retest'. The questionnaire had a validity of .93 , while $72.73 \%$ and $27.27 \%$ of the items had an excellent reliability and good reliability, respectively. This allows us to conclude that this questionnaire meets its objectives and will serve to know the techniques and pressure of the cuff that health professionals in Chile use for the management of adult patients with an artificial airway.
\end{abstract}

Keywords: airway management, tracheostomy, intubation endotracheal, cuff, questionnaires, Chile. 


\section{Introducción}

El balón o 'cuff' que poseen los tubos endotraqueales y algunos tipos de cánulas de traqueostomías tiene como objetivo sellar la vía aérea durante la ventilación mecánica y evitar el riesgo de aspiración (Calder \& Pearce, 2010; J. Dorsch \& Dorsch, 2008). Su manejo adecuado contribuye a evitar el daño traqueal cuando el 'cuff' se encuentra a presiones de insuflación elevadas y el desarrollo de neumonía aspirativa asociado a presiones inferiores. Las presiones de insuflación del 'cuff' pueden ser administradas mediante técnicas objetivas las que utilizan valores en centímetros de agua $(\mathrm{cm} \mathrm{H2O})$ o en milímetros de mercurio $(\mathrm{mm} \mathrm{Hg})$, o con técnicas subjetivas, las que no utilizan valores referenciales (Félix-Ruiz, López-Urbina, \& Carrillo-Torres, 2014). Actualmente, la evidencia internacional recomienda diferentes rangos de presión a utilizar en usuarios con vía aérea artificial (Bodenham et al., 2014; Credland, 2015; Das \& Kumar, 2015; De Leyn et al., 2007; Hess, 2005; Russell \& Matta, 2004; Sole et al., 2011), siendo el más alto reportado entre 25 y $35 \mathrm{~cm} \mathrm{H} 2 \mathrm{O}$, mientras que el más bajo entre 15 y $25 \mathrm{~cm} \mathrm{H2O}$. En nuestro país, no existe una guía clínica ni directriz ministerial sobre el manejo de usuarios con vía aérea artificial. Además, solo algunas instituciones sanitarias han desarrollado protocolos propios recomendando diferentes rangos de presión, en donde la máxima recomendada equivale a $30 \mathrm{~mm} \mathrm{Hg}$ (alrededor de 40 cm H2O) y la mínima a 22 cm H2O (Hospital de Castro, 2012; Hospital de Iquique, 2015; Hospital de la Florida, 2014; Hospital de Talca, 2010; Hospital Santiago Oriente, 2013). Cabe destacar que varias de las sugerencias mencionadas tanto por la literatura internacional como por los documentos nacionales han sido basadas en referencias de artículos primarios en cuyas investigaciones se utilizó población animal. Además, si bien existen las recomendaciones de ciertas instituciones sanitarias, actualmente no se cuenta con un catastro nacional de los rangos utilizados por los diferentes profesionales de la salud.

Los antecedentes disponibles indican que en Chile los profesionales de la salud no están utilizando los mismos criterios clínicos para la adecuada atención de usuarios con vía aérea artificial, situación que podría revertirse con el desarrollo de una referencia única nacional que contemple recomendaciones y sugerencias ministeriales basadas en evidencia científica.

En un estudio previo a este trabajo (Rosales, 2019), se llevó a cabo una revisión literaria para identificar el rango adecuado de presión a utilizar en los 'cuffs' de usuarios con vía aérea artificial. Dicha revisión permitió concluir que en población humana debiese ser entre 20 y $30 \mathrm{~cm} \mathrm{H} 20$ (Rosales, 2019). EI resultado anterior concuerda con el rango propuesto por Maldonado, Fuentes, Riquelme, Sáez, \& Villarroel (2018), miembros de la Sociedad Chilena de Medicina Intensiva. El presente trabajo corresponde a la segunda parte del estudio referido y tiene como objetivo crear un cuestionario válido y confiable sobre las técnicas y el manejo de la presión del 'cuff,' utilizados en usuarios adultos con vía aérea artificial por profesionales de la salud en Chile. La creación de este cuestionario permitirá a futuro conocer cuáles son las técnicas y los rangos de presión del 'cuff' utilizados en Chile por los profesionales de la salud, para el manejo de usuarios con vía aérea artificial. 


\section{Metodología}

\section{Diseño}

Este trabajo corresponde a un método mixto cualitativo - cuantitativo CUAL -> CUAN tipo VIII secuencial, descriptivo y longitudinal (Pereira, 2011). Dicho método fue usado para crear un cuestionario sobre el manejo de la presión del 'cuff' en pacientes adultos con vía aérea artificial por profesionales de la salud en Chile. Es cualitativo ya que dentro de las fases del estudio un grupo de hablantes nativos de español y un grupo de expertos en el manejo de usuarios con vía aérea artificial fueron consultados. Al primer grupo se le solicitó analizar la traducción del instrumento del inglés (idioma en el que fue creado) al español junto con proponer posibles modificaciones. El segundo grupo analizó el contenido del instrumento y entregó recomendaciones para mejorarlo. Es cuantitativo porque el cuestionario fue analizado mediante un software estadístico para conocer su validez de contenido y confiabilidad. También es cuantitativo porque se utilizaron exclusivamente datos categóricos y continuos. Finalmente, es longitudinal debido a que el cuestionario fue testeado en tiempos diferentes para medir su validez de contenido y su confiabilidad.

\section{Participantes}

El grupo de participantes en las distintas etapas estuvo compuesto por enfermeros, kinesiólogos y fonoaudiólogos residentes en Chile con experiencia en el manejo de usuarios con vía aérea artificial. Ellos se dividieron en dos grupos por conveniencia.
El primero estuvo compuesto por diez expertos chilenos, los que participaron en la fase de la medición de validez del instrumento (fase B) y tenían un mínimo de cinco años de experiencia laboral. La mitad de ellos trabajaban en instituciones de salud públicas, mientras que la otra mitad en privadas. Siete expertos trabajaban en Santiago y tres en regiones. Además, del total de este grupo, siete de ellos tenían experiencia activa trabajando en universidades chilenas como docentes y/o tutores de práctica profesional en las instituciones de salud donde se desempeñaban. La Tabla 1 muestra la descripción de los expertos voluntarios de la fase $B$.

El segundo grupo participó en la fase de evaluación de la implementación del instrumento y en la medición de la confiabilidad (fase C). Este grupo estaba compuesto por treinta y tres profesionales de salud que poseían experiencia clínica en el manejo de pacientes con vía aérea artificial en instituciones de salud públicas o privadas. En una primera instancia, un total de cuarenta y tres voluntarios declararon su interés en participar. Solo treinta y siete de los cuarenta y tres voluntarios dieron su consentimiento y completaron la encuesta satisfactoriamente en el 'Tiempo 1'. A dicho grupo se le envió el cuestionario durante el 'Tiempo 2', donde treinta y tres participantes respondieron el instrumento de forma completa (seis enfermeros, once kinesiólogos y dieciséis fonoaudiólogos), quienes conformaron la muestra definitiva para la medición de la confiabilidad. Tomando en cuenta el total de voluntarios iniciales versus los finales, la tasa de respuesta fue de un $77 \%$. 

en Chile

Tabla 1

Descripción de los expertos voluntarios de la fase $B$.

\begin{tabular}{llllc}
\hline Experto & Profesión & Institución de salud de desempeño & Experiencia docente & Ciudad \\
\hline 1 & Kinesiólogo & Pública & Sí & Santiago \\
2 & Kinesiólogo & Pública & Sí & Curicó \\
3 & Kinesiólogo & Privada & Sí & Santiago \\
4 & Fonoaudiólogo & Privada & Sí & Santiago \\
5 & Fonoaudiólogo & Pública & Sí & Rancagua \\
6 & Fonoaudiólogo & Pública & Sí & Curicó \\
7 & Enfermero & Privada & No & Santiago \\
8 & Enfermero & Privada & Sí & Santiago \\
9 & Enfermero & Pública & No & Santiago \\
10 & Enfermero & Privada & No & Santiago \\
\hline
\end{tabular}

\section{Instrumentos}

El cuestionario fue desarrollado utilizando literatura relacionada con la creación de cuestionarios (Hulley, Cummings, Browner, Grady, \& Newman, 2013; Oppenheim, 2005; Ross, 2005; Tools4dev, 2014).

Las preguntas se caracterizaron por ser cortas, cerradas y específicas. Las palabras utilizadas fueron familiares, se usó un formato de opción múltiple y completación de valores numéricos. Ello arrojó tanto datos continuos como categóricos.

Para medir la validez del instrumento, se elaboró un formulario de Índice de Validez de Contenido (Content Validity Index, ${ }^{\prime} \mathrm{CVI}$ '). Para medir la confiabilidad, se desarrolló un cuestionario en línea de modalidad auto administrada mediante la plataforma 'iSurvey', asociada a la Universidad de Southampton. La modalidad en línea fue seleccionada debido a la rapidez en la administración, tanto la recolección como el análisis de datos, costo y especificidad. Así, se evitó posibles errores humanos durante el traspaso de la información (Hulley et al., 2013; Oppenheim, 2005; Polit \& Tatano, 2006; Ross, 2005).

\section{Procedimientos}

Se llevaron a cabo tres fases, las que se detallan a continuación.

En la fase A se desarrolló un borrador del cuestionario en inglés, el que en total contenía veintiocho preguntas. Dicho cuestionario fue creado en inglés debido a que el proyecto se llevó a cabo en una institución del Reino Unido, por lo que parte del equipo de investigación era anglófono.

Luego, el borrador fue traducido al español y enviado a tres profesores hablantes nativos de español asociados a la Facultad de Ciencias de la Salud de la Universidad de Southampton, con el fin de juzgar 
la traducción y dar sus opiniones en reuniones conjuntas para posibles modificaciones y posterior aprobación tras su mejora.

En la fase B un grupo de expertos chilenos analizó el borrador en español. El grupo estaba compuesto por profesionales clínicos o profesores universitarios con al menos 5 años de experiencia en el manejo de usuarios con vía aérea artificial (cuatro enfermeros, tres kinesiólogos y tres fonoaudiólogos de instituciones de salud pública y privada). Estos expertos fueron consultados para identificar posibles problemas relacionados al contenido del cuestionario y para medir su validez. La relación del investigador con los expertos se estableció exclusivamente para estos fines. Se envió un correo electrónico invitándolos a participar, explicando el proyecto y la importancia del desarrollo de un cuestionario en el área, junto a la importancia de su participación voluntaria. Luego de confirmar la participación, los expertos recibieron el borrador del cuestionario en español junto a su respectivo flujograma mediante correo electrónico (ver Figura 1). Se solicitó a cada experto entregar información en detalle con sus recomendaciones. Ello, con el fin de que el investigador pudiera revisar los ítems que ellos consideraran necesarios modificar. Se estimó un plazo de diez días para realizar el proceso. El investigador se reunió con cada uno de los expertos para aclarar posibles dudas previo del envío de sus recomendaciones.

Tras la recepción de los comentarios vía correo electrónico, el borrador en español fue rediseñado con la nueva información. Posteriormente, un nuevo correo electrónico se envio con el borrador rediseñado y el formulario de 'CVI' para medir la validez de contendido. Lo anterior se hizo a través del juicio del nivel de relevancia de cada ítem. Los ítems fueron categorizados en una escala de ' 1 ' a ' 4 ' desde 'no relevante' a 'altamente relevante'. Se entregaron diez días extras como plazo para completar el formulario.

En la fase C, para identificar posibles problemas sobre la implementación del cuestionario y medir su confiabilidad, fue aplicado (piloto) dos veces ('Tiempo 1 ' y 'Tiempo 2') a 33 profesionales de salud en Chile (Credland, 2015). El método para reclutar a los voluntarios fue a través de avisos a los colegios profesionales y en 'Linkedln'. Luego que los voluntarios confirmaron su participación, sus correos electrónicos se requirieron para ser añadidos de forma manual a 'iSurvey', plataforma la cual envió a los voluntarios el enlace del cuestionario en el 'Tiempo 1' y 'Tiempo 2'. El instrumento fue completado por los participantes durante los primeros diez días después de recibir el enlace por correo electrónico en el 'Tiempo 1' y en el 'Tiempo 2'. La diferencia entre la aplicación del cuestionario entre ambos periodos fue de treinta días. Posteriormente, el cuestionario fue testeado a través de la prueba 'test-retest').

\section{Consideraciones éticas}

El protocolo de este proyecto fue revisado por el comité de ética de la Universidad de Southampton, el que fue aprobado e identificado con el número 41358.A1. Se presentó un consentimiento informado a los participantes al momento de abrir el enlace del cuestionario. Dicho consentimiento contenía información sobre anonimato, uso, codificación y 
eliminación de los datos tras su utilización. Junto a lo anterior, también se incluyen en el consentimiento las políticas de protección de datos de la University of Southampton (2018) y la regulación de protección de datos de la Unión Europea (Intersoft Consulting, 2018). Aquellos participantes quienes no dieron su consentimiento no tuvieron acceso al instrumento.

\section{Análisis de datos}

Para medir la validez de contenido, se utilizó un formulario de 'CVI' en la fase $\mathrm{B}$, que fue dividido en la medición de la validez por ítem ('I-CVI') y total ('S$\left.\mathrm{CVI}^{\prime}\right)$. Para ello, los expertos juzgaron cada ítem del cuestionario en relación con las siguientes categorías: 1- 'no relevante' / 2- 'algo relevante' / 3- 'relevante' / 4- 'altamente relevante' (Chiwaridzo et al., 2017; Oppenheim, 2005; Polit \& Tatano, 2006). Posteriormente, las categorías 'no relevante' y 'algo relevante' fueron cambiadas por el número ' 0 ', mientras que las categorías 'relevante' y 'altamente relevante' fueron modificadas por el número ' 1 '. Todos los números ' 0 ' fueron interpretados como 'ítems no relevantes' mientras que los números ' 1 ' como 'ítems relevantes'.

Para calcular 'I-CVI', se promedió cada ítem según el número de expertos, siendo el resultado interpretado como el nivel de acuerdo entre expertos. Cada 'I-CVI' fue considerado válido con promedios iguales o superiores a 0,78 (Polit \& Tatano, 2006). La totalidad de los ítems en que sus promedios fueron menores a 0,78 se eliminaron automáticamente.

Posteriormente, se calculó el 'S-CVI' usando la técnica de 'Scale Content Validity Index Average' en el que los 'I-CVI' fueron promediados. El criterio estándar usado para el cálculo de 'S-CVI' fue de un valor igual o mayor a 0,90 (Polit \& Tatano, 2006). La confiabilidad fue testeada mediante el test de confiabilidad 'test-retest' usando técnicas estadísticas mediante el software 'Statistical Package for the Social Sciences' ('SPSS') considerando un intervalo de confianza ('I.C.') de 95\% y un valor p menor de 0,05.

Dado que el cuestionario no era una escala en la que se calcula un puntaje total final, y además contenía datos categóricos y continuos, el análisis fue realizado por ítem, usando la prueba de Correlación Intraclase ('ICC') (Singh et al., 2011). Este análisis contenía los siguientes coeficientes: 'excelente' (igual o mayor a 0,81); 'bueno' (desde 0,61 a 0,80); 'moderado' (desde 0,41 a 0,60) y 'pobre' (igual o menor que 0,40). El método 'ICC' para medir la confiabilidad intra-evaluador se realizó a través del análisis 'two-way mixed effects, absolute agreement' (Koo \& Li, 2016; Singh et al., 2011) utilizando el software 'SPSS'. El 'ICC' fue exclusivamente usado para ítems con datos continuos y categóricos de tipo no dicotómicos (Singh et al., 2011). Para el cálculo de los ítems categóricos dicotómicos, se utilizó el porcentaje de acuerdo ('PA') con la siguiente categoría: 'excelente' (igual o mayor a 90\%); 'buena' (desde 75 a 89\%), 'moderada' (desde 60 a 74\%), y 'pobre' (menor a 60\%) (Hulley et al., 2013). Para el cálculo de 'ICC' se considera que la existencia de variabilidad es importante. Las preguntas con nula variabilidad fueron analizadas usando 'PA'.

\section{Resultados}

A continuación, se presentan los resultados de las distintas fases. 


\section{Fase A: borrador del cuestionario}

Dos de los hablantes de español nativos recomendaron mínimos cambios en la traducción de algunas palabras del borrador desde el inglés al español. Luego, el instrumento fue modificado en relación con sus sugerencias y aprobado por ellos.

\section{Fase B: validez de contenido}

Luego de modificar el borrador en español de acuerdo con las sugerencias de los expertos, ellos completaron el formulario de 'CVI' (Chiwaridzo et al., 2017; Oppenheim, 2005; Polit \& Tatano, 2006) para juzgar cada ítem del cuestionario. El 'S-CVI' fue calculado utilizando exclusivamente resultados de 'ICVI' iguales o mayores a 0,78 (Polit \& Tatano, 2006). Los ítems '10'; ' 12'; ' 15’; '17' (relacionados a la forma en que los participantes adquirieron el conocimiento sobre el uso de técnicas subjetivas); '21' (la elección entre técnicas objetivas versus subjetivas): '22' (género del participante); y '28' (región donde el participante trabajaba) obtuvieron 'I-CVI' menores a 0,78 . Dichos resultados fueron 0,$70 ; 0,70 ; 0,60 ; 0,50$; 0,$70 ; 0,40$ y 0,40 , respectivamente. Por esta razón, fueron eliminados. El cuestionario final obtuvo un 'S$\mathrm{CVI}^{\prime}$ de 0,93 el cual fue considerado como un puntaje aceptable para validar el contenido del instrumento (Polit \& Tatano, 2006).

El cuestionario definitivo contuvo veintiún preguntas de las cuales siete se relacionaron al uso de técnicas objetivas, seis al uso de técnicas subjetivas, tres al uso de ambas técnicas y cinco a información de los participantes (ver Figura 1). El ítem, '7' contenía dos respuestas de tipo numérica, por lo que para su análisis fue dividido en dos: ' $7 a$ ' y '7b'. La Tabla 2 muestra el resultado final del proceso de validación de contenido del instrumento en donde se detallan los ítems con 'I-CVI' igual o mayor a 0,78 con los que se calculó el 'S-CVI'. 


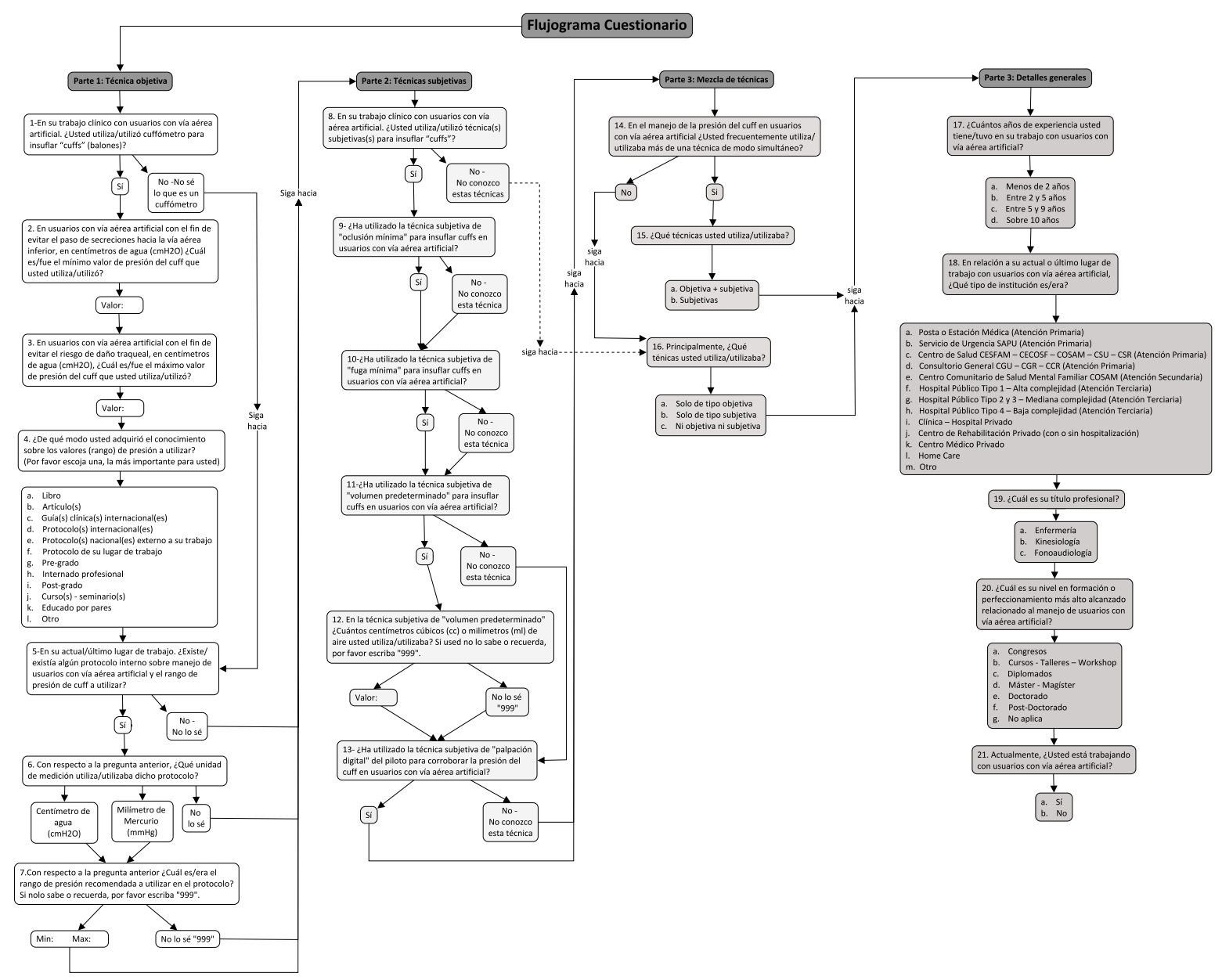

Gráfico 1. Flujograma del cuestionario definitivo luego de la validación por parte del grupo de expertos. 
Tabla 2

Resumen del proceso de validación de contenido del cuestionario luego del juicio de cada experto (E), donde los números ' 1 ' son items 'relevantes' mientras que los números ' 0 ' 'no relevantes'.

\begin{tabular}{|c|c|c|c|c|c|c|c|c|c|c|c|}
\hline Ítem & E1 & E2 & E3 & E4 & E5 & E6 & E7 & E8 & E9 & E10 & Total 'I-CVI' \\
\hline 1 & 1 & 1 & 1 & 1 & 1 & 1 & 1 & 1 & 1 & 1 & 1 \\
\hline 2 & 1 & 1 & 1 & 1 & 1 & 1 & 1 & 1 & 1 & 1 & 1 \\
\hline 3 & 1 & 1 & 1 & 1 & 1 & 1 & 1 & 1 & 1 & 1 & 1 \\
\hline 4 & 1 & 1 & 0 & 1 & 1 & 1 & 1 & 1 & 1 & 1 & 0,90 \\
\hline 5 & 1 & 1 & 1 & 1 & 1 & 1 & 1 & 1 & 1 & 1 & 1 \\
\hline 6 & 1 & 1 & 1 & 1 & 1 & 1 & 1 & 1 & 1 & 1 & 1 \\
\hline 7 & 1 & 1 & 1 & 1 & 1 & 1 & 1 & 1 & 1 & 1 & 1 \\
\hline 8 & 1 & 1 & 1 & 1 & 1 & 0 & 1 & 1 & 1 & 0 & 0,80 \\
\hline 9 & 1 & 1 & 1 & 1 & 1 & 1 & 1 & 1 & 1 & 0 & 0,90 \\
\hline 10 & 1 & 1 & 1 & 1 & 1 & 1 & 1 & 1 & 1 & 0 & 0,90 \\
\hline 11 & 1 & 1 & 1 & 1 & 1 & 1 & 1 & 1 & 1 & 0 & 0,90 \\
\hline 12 & 1 & 1 & 1 & 1 & 1 & 1 & 1 & 1 & 1 & 0 & 0,90 \\
\hline 13 & 1 & 1 & 1 & 1 & 1 & 0 & 1 & 1 & 1 & 0 & 0,80 \\
\hline 14 & 1 & 1 & 1 & 1 & 1 & 1 & 1 & 1 & 1 & 1 & 1 \\
\hline 15 & 1 & 1 & 1 & 1 & 1 & 1 & 1 & 1 & 1 & 1 & 1 \\
\hline 16 & 1 & 1 & 1 & 1 & 1 & 1 & 1 & 1 & 1 & 1 & 1 \\
\hline 17 & 1 & 1 & 1 & 1 & 1 & 1 & 1 & 1 & 1 & 1 & 1 \\
\hline 18 & 1 & 1 & 1 & 0 & 1 & 1 & 1 & 1 & 0 & 1 & 0,80 \\
\hline 19 & 1 & 1 & 1 & 1 & 1 & 1 & 1 & 1 & 1 & 0 & 0,90 \\
\hline 20 & 1 & 1 & 1 & 0 & 1 & 1 & 1 & 1 & 1 & 0 & 0,80 \\
\hline 21 & 1 & 1 & 1 & 1 & 1 & 1 & 1 & 1 & 1 & 1 & 1 \\
\hline Promedio & 1 & 1 & 0,95 & 0,90 & 1 & 0,90 & 1 & 1 & 0,95 & 0,62 & 0,93 ('S-CVI') \\
\hline
\end{tabular}

\section{Fase C: confiabilidad 'test-retest'}

La Tabla 3 resume el análisis usando el 'ICC' para ítems continuos y categóricos no dicotómicos, y el 'PA' para los ítems categóricos y categóricos no dicotómicos con nula variabilidad. El análisis muestra que dieciséis ítems lograron una confiabilidad 'excelente' (72,73\%) mientras seis lograron 'buena' confiabilidad $(27,27 \%)$. 
Cuestionario sobre el uso de técnicas y manejo de presión del cuff en usuarios adultos con vía aérea artificial por parte de profesionales de salud en Chile

Tabla 3

Análisis para calcular la confiabilidad 'test-retest' mediante 'ICC' y 'PA' por ítem, y sus respectivas clasificaciones (Clas): $E$ ('excelente'), B ('bueno'), $M$ ('moderado') y $P$ ('pobre').

\begin{tabular}{|c|c|c|c|c|c|c|}
\hline Dato & Ítem & 'ICC' & Clas. & Valor $p$ & 'PA' & Clas. \\
\hline & A. Uso de técnicas objetivas & & & & & \\
\hline Categórico & 1.- Uso de cuffómetro* & & & & 93,94 & $\mathrm{E}$ \\
\hline Continuo & 2- Presión mínima de 'cuff' usada & 0,89 & $\mathrm{E}$ & 0,01 & & \\
\hline Continuo & 3- Presión máxima de 'cuff' usada & 0,90 & $\mathrm{E}$ & 0,01 & & \\
\hline Categórico & 4- Adquisición del conocimiento & 0,70 & B & 0,01 & & \\
\hline Categórico & 5- Protocolo en lugar de trabajo* & & & & 81,82 & B \\
\hline Categórico & 6- Unidad de medida (protocolo)* & & & & 100 & $\mathrm{E}$ \\
\hline Continuo & 7a- Presión mínima del 'cuff'(protocolo) & 0,96 & $\mathrm{E}$ & 0,01 & & \\
\hline \multirow[t]{2}{*}{ Continuo } & 7b- Presión máxima del 'cuff'(protocolo) & 0,99 & $\mathrm{E}$ & 0,01 & & \\
\hline & B. Uso de técnicas subjetivas & & & & & \\
\hline Categórico & 8-Técnicas subjetivas* & & & & 93,94 & $\mathrm{E}$ \\
\hline Categórico & 9- Técnica de oclusión mínima* & & & & 86,36 & $B$ \\
\hline Categórico & 10- Técnica de fuga mínima* & & & & 81,82 & $B$ \\
\hline Categórico & 11- Técnica volumen predeterminado* & & & & 77,27 & B \\
\hline Continuo & 12- Volumen predeterminado usado & 1 & $\mathrm{E}$ & 0,01 & & \\
\hline \multirow[t]{2}{*}{ Categórico } & 13- Técnica de palpación digital del 'cuff'* & & & & 90,48 & $E$ \\
\hline & C. Mezcla de técnicas & & & & & \\
\hline Categórico & 14- Uso de ambas técnicas* & & & & 84,85 & B \\
\hline Categórico & 15- Mezcla de técnicas* & & & & 94,12 & $\mathrm{E}$ \\
\hline \multirow[t]{2}{*}{ Categórico } & 16- Principal técnica usada* & & & & 100 & $\mathrm{E}$ \\
\hline & D. Información de los participantes & & & & & \\
\hline Categórico & 17-Años de experiencia en el área & 0,99 & $\mathrm{E}$ & 0,01 & & \\
\hline Categórico & 18- Lugar de trabajo & 0,95 & $\mathrm{E}$ & 0,01 & & \\
\hline Categórico & 19- Título profesional** & & & & 100 & $\mathrm{E}$ \\
\hline Categórico & 20- Nivel de entrenamiento más alto & 0,89 & $\mathrm{E}$ & 0,01 & & \\
\hline Categórico & 21- Trabajo actual en el área13 & & & & 100 & $E$ \\
\hline
\end{tabular}

* Items dicotómicos en el que sus ' $P A^{\prime}$ ' fueron usados.

** Un ítem no dicotómico con nula variabilidad en el que su 'PA' fue usado. 
El tiempo total en el que los participantes demoraron en responder fue analizado de forma descriptiva mediante 'SPSS' en el 'Tiempo 1' y en el 'Tiempo 2' junto al tiempo total de completación de la herramienta en segundos (s). Los participantes con valores atípicos no fueron incluidos en el análisis. Por ello, se analizaron veinte participantes en donde los datos estuvieron normalmente distribuidos con valores de asimetría de 0,92 y 1,90 respectivamente, considerados dentro de los rangos normales de asimetría (Dancey, Reidy, \& Rowe, 2012). Debido a ello, se utilizó la media para el reporte. El promedio total en el que los participantes demoraron en responder el cuestionario en el 'Tiempo 1' fue de 679 s mientras que en el 'Tiempo 2' fue de 443 s. Dichos promedios fueron equivalentes a 11 minutos $19 \mathrm{~s}$ y a 7 minutos 23 s, respectivamente. En relación con los treinta y tres participantes, quienes respondieron el cuestionario en las dos ocasiones, la diferencia que ellos recibieron entre el enlace y su completación final fue de 4,71 días en el 'Tiempo 1' y de 4,13 días en el 'Tiempo 2'.

\section{Discusión}

Este trabajo tuvo por objetivo crear un cuestionario válido y confiable sobre las técnicas y el manejo de la presión del 'cuff' en usuarios adultos con vía aérea artificial por profesionales de la salud en Chile.

El cuestionario tuvo una validez de contenido aceptable $(0,93)$ en relación con la literatura (Polit \& Tatano, 2006). Como fue reportado, algunos ítems del borrador fueron eliminados ya que sus respectivos 'ICVI' fueron menores a 0,78 . Ello a pesar de que previo al juicio de expertos, el equipo investigador consideró que tenían relevancia para obtener información sobre las técnicas subjetivas y la adquisición de dicho conocimiento.

En términos generales, un alto porcentaje de ítems tuvo 'excelente' confiabilidad. No hubo ítems con categorías 'moderada' ni 'pobre'. Por esta razón, el cuestionario será útil para futuras administraciones. Al respecto, cabe señalar que el 'test-retest' tiene algunas ventajas y desventajas. Es posible que algunas respuestas hayan estado influenciadas por la memoria de los participantes, en la que posibilitaron responder la misma información en ambas aplicaciones del cuestionario, lo que pudo incrementar los coeficientes de confiabilidad (Hulley et al., 2013). Sin embargo, es importante destacar que el cuestionario tuvo un lapso de treinta días de diferencia entre ambas aplicaciones, lo que es sugerido según la literatura para los instrumentos que miden variables estables (Miller \& Salkind, 2002). Debido a esto, se evitó el problema de los coeficientes altamente confiables relacionados a lapsos de tiempo cortos, versus coeficiente con baja confiabilidad relacionados a lapsos de tiempos largos (Carvajal, Centeno, Watson, Martínez, \& Rubiales, 2011).

Un ítem que es importante discutir es el número '12' el cual tuvo una 'excelente' confiabilidad mediante 'ICC'. Este ítem está relacionado a la técnica de volumen predeterminado la cual es una técnica en el que el 'cuff' es insuflado usando un volumen particular de aire con jeringa. Llama la atención este nivel de confiabilidad, dado que no se encontraron estudios previos relevantes, ni literatura que recomiende un volumen de aire específico a ser utilizado. Habría sido importante conocer cómo los 
clínicos aprendieron el volumen de aire a utilizar, considerando que los pacientes tienen diferencias anatómicas y diferentes tipos de tubos (traqueostomías versus tubos endotraqueales; marca de los tubos; número de cánulas, entre otras). Por ello, no es razonable que el volumen predeterminado de aire pueda ser usado para insuflar 'cuffs'.

Por otra parte, algunos ítems presentaron una confiabilidad 'buena' y no 'excelente'. Esto puede deberse a varias razones. El hecho de que los rasgos cambian en el tiempo, independientemente del tipo de datos, puede ser una de las razones de que los participantes respondieron diferente información en algunas preguntas. Es posible que los factores que influyeron en este cambio estuvieran relacionados a que los participantes buscaran información más apropiada tras responder el primer cuestionario, para usarla durante la segunda aplicación. Otra posible razón es que luego de leer el cuestionario en la primera aplicación, durante la práctica clínica pudieron usar técnicas que no utilizaban y que aprendieron en la primera administración. También puede haber disminuido el interés en responder el instrumento la segunda vez, originando algunas respuestas al azar (Carvajal et al., 2011).

Cabe señalarque la tasa de respuesta general del cuestionario fue de un $77 \%$. Ello es mayor al porcentaje descrito por la literatura para este tipo de herramientas en línea (Fincham, 2008), donde se menciona que normalmente puede ser alrededor del $70 \%$, cuando métodos de alcance son utilizados. Esta tasa se pudo asociar a dos factores que posiblemente incrementan la respuesta de los participantes (Carvajal et al., 2011; Miller \& Salkind, 2002; Saleh \& Bista, 2017) como aquellos que tengan relación al tema principal de investigación y el envío de recordatorios. Además, 4,71 y 4,13 fueron los días promedio en el que los participantes respondieron el instrumento entre el día que recibieron el enlace del cuestionario y su completación, tanto en el 'Tiempo 1 ' como en el 'Tiempo 2'. Dichos días fueron menores a los 7,6 reportados por la literatura para instrumentos en línea (Saleh \& Bista, 2017).

Como limitaciones de este trabajo, se debe mencionar que durante la fase A la traducción del instrumento fue llevada a cabo solo por el investigador principal. Además, no se pudieron aplicar criterios extras para la selección de los hablantes nativos de español debido al limitado número de profesores hablantes nativos de español contratados en la Facultad de Ciencias de la Salud de la Universidad de Southampton. Durante la fase B, si bien se efectuaron visitas a cada experto para aclarar dudas, no fue posible reunir a todo el equipo. Ello con el fin de determinar en conjunto las modificaciones a realizar al instrumento previo a la aplicación del 'CVI' debido a que algunos de los expertos vivían en ciudades fuera de la Región Metropolitana. Por ello, el borrador fue modificado considerando todas las opiniones de los expertos, que luego fue enviado para ser evaluado individualmente.

A modo de reflexión, cabe señalar que es necesaria una actualización total de la información relacionada al riesgo de aspiración y de daño traqueal a través de nuevas investigaciones en población humana, con tecnología actual. Además, es fundamental que los distintos países revisen sus guías clínicas, protocolos y evidencia acerca del rango de presión del 'cuff' a utilizar, con el fin de asegurar un manejo seguro en usuarios con vía aérea artificial. 
Agradecimiento: el autor agradece a la Comisión Nacional de Investigación Científica y Tecnológica CONICYT que otorgó la Beca de Magíster en el extranjero periodo 2017-2018 del 'Master in Clinical and Health Research' en la Universidad de Southampton, Reino Unido; a las Doctoras Bronagh Walsh, líder del programa, y Dawn-Marie Walker, supervisora del proyecto de investigación; al grupo de expertos y profesionales voluntarios; a Patricia Acosta y Talia Emmanuel por los meses de estudios; y finalmente a Tiago Azevedo quien formó parte importante de todo este proceso académico.

\section{Referencias}

Bodenham, A., Bonner, S., Branch, F., Dawson, D., Morgan, P., McGrath, B., \& Mackenzie, S. (2014). Standards for the care of adult patients with a temporary Tracheostomy. Recuperado https://www.theawsomecourse.co.uk/ICS/ICS\%20Tracheo stomy\%20standards\%20(2014).pdf

Calder, I., \& Pearce, A. (2010). Core Topics in Airway Management. Recuperado de https://books.google.cl/books?id=30lajBLGnNMC\&printsec $=$ frontcover\&dq=Core+Topics+in+Airway+Management\&hl =es-

419\&sa=X\&ved=0ahUKEwj5267pz43mAhUGHrkGHTrDC_Y Q6AEILDAA\#v=onepage $\& q=$ Core\%20Topics\%20in\%20Airw ay\%20Management\&f=false

Carvajal, A., Centeno, C., Watson, R., Martínez, M., \& Rubiales, A. S. (2011). How is an instrument for measuring health to be validated? Anales Del Sistema Sanitario De Navarra, 34(1), 63-72. https://doi.org/10.4321/s113766272011000100007

Chiwaridzo, M., Chikasha, T. N., Naidoo, N., Dambi, J. M., Tadyanemhandu, C., Munambah, N., \& Chizanga, P. T. (2017). Content validity and test-retest reliability of a low back pain questionnaire in Zimbabwean adolescents. Archives of Physiotherapy, 7, 3. https://doi.org/10.1186/s40945-017-0031-y

Credland, N. (2015). How to measure tracheostomy tube cuff pressure. Nursing Standard, 30(5), 36-38. https://doi.org/10.7748/ns.30.5.36.e9495

Dancey, C., Reidy, J., \& Rowe, R. (2012). Statistics for the Health Sciences: A Non-Mathematical Introduction. SAGE.

Das, S., \& Kumar, P. (2015). Comparison of minimal leak test and manual cuff pressure measurement technique method for inflating the endotracheal tube cuff. https://doi.org/10.5958/2394-4994.2015.00002.5

De Leyn, P., Bedert, L., Delcroix, M., Depuydt, P., Lauwers, G., Sokolov, Y., ... Belgian Association of Pneumology and Belgian Association of Cardiothoracic Surgery. (2007). Tracheotomy: Clinical review and guidelines. European Journal of Cardio-Thoracic Surgery: Official Journal of the European Association for Cardio-Thoracic Surgery, 32(3), 412-421. https://doi.org/10.1016/j.ejcts.2007.05.018

Dorsch, J., \& Dorsch, S. (2008). Understanding Anesthesia Equipment (5a $\quad$ ed.). Recuperado de https://books.google.cl/books?id=Itqdx2lyRxcC\&printsec=f rontcover\&dq=Understanding+Anesthesia+Equipment\&hl= es-419\&sa=X\&ved=0ahUKEwj_94-

SOY3mAhUgl7kGHei3BssQ6AEILDAA\#v=onepage \&q=Under standing\%20Anesthesia\%20Equipment $\& f=$ false

Félix-Ruiz, R., López-Urbina, D. M., \& Carrillo-Torres, O. (2014). Evaluar la precisión de las técnicas subjetivas de insuflación del globo endotraqueal. Revista Mexicana de Anestesiología, 37(2), 71-76. Recuperado de https://www.medigraphic.com/cgi-

bin/new/resumen.cgi?IDARTICULO=50130

Fincham, J. E. (2008). Response Rates and Responsiveness for Surveys, Standards, and the Journal. American Journal of Pharmaceutical Education, 72(2). Recuperado de https://www.ncbi.nlm.nih.gov/pmc/articles/PMC2384218/

Hess, D. R. (2005). Tracheostomy tubes and related appliances. Respiratory Care, 50(4), 497-510.

Hospital de Castro. (2012). Protocolo de manejo de enfermería en traqueostomía y tubo endotraqueal en el adulto. Recuperado de http://biblioteca.hospitalcastro.gov.cl/index.php?option=c om_phocadownload\&view=file\&id=342: protocolo-demanejo-de-enfermeria-en-traqueostomia-y-tuboendotraqueal $\&$ Itemid $=77 \&$ start $=48$

Hospital de Iquique. (2015). Protocolo manejo de tubo endotraqueal y traqueostomía. Recuperado de http://www.hospitaliquique.cl/images/PCl/GCL-1.2.3- 


\section{Manejo-TET.pdf}

Hospital de la Florida. (2014). Protocolo de manejo de traqueostomía y tubo endotraqueal del hospital de la Florida. Recuperado de http://hospitaldeflorida.dssc.cl/wpcontent/uploads/2016/02/GCL\%201.2\%20TRAQUESTOMIA .pdf

Hospital de Talca. (2010). Normas y Protocolos de Prevención de Infecciones de Vía Aérea. Recuperado de http://www.hospitaldetalca.cl/adicional/documentos/Viaa erea.pdf

Hospital Santiago Oriente. (2013). Protocolo manejo de enfermería en traqueostomía y tubo endotraqueal en adulto. Recuperado de http://200.72.129.100/calidad/archivo1/Tubo\%20Endotra queal\%20Adulto\%20-\%20GCL\%201.2_v.4.pdf

Hulley, S. B., Cummings, S. R., Browner, W. S., Grady, D. G., \& Newman, T. B. (2013). Designing Clinical Research (4ta ed.). Philadelphia: Lippincott Williams\&Wilki.

Intersoft Consulting. (2018). European Union General Data Protection Regulation (GDRP). Recuperado de https://gdprinfo.eu

Koo, T. K., \& Li, M. Y. (2016). A Guideline of Selecting and Reporting Intraclass Correlation Coefficients for Reliability Research. Journal of Chiropractic Medicine, 15(2), 155-163. https://doi.org/10.1016/j.jcm.2016.02.012

Maldonado, E., Fuentes, I., Riquelme, M. L., Sáez, M., \& Villarroel, E. (2018). Documento de Consenso: Prevención de Neumonía Asociada a Ventilación Mecánica del Adulto. Revista Chilena de Medicina Intensiva, 33(1), 15-28.

Miller, D., \& Salkind, N. (2002). Handbook of Research Design and Social Measurement (6 $6^{\mathrm{a}}$ ed.). Recuperado de https://us.sagepub.com/en-us/nam/handbook-ofresearch-design-and-social-measurement/book10296

Oppenheim, A. N. (2005). Questionnaire Design, Interviewing and Attitude Measurement. Bloomsbury Academic.

Pereira, Z. (2011). Los diseños de método mixto en la investigación en educación: Una experiencia concreta. Revista Electrónica Educare, XV(1), 15-29. Recuperado de https://www.redalyc.org/articulo.oa?id=194118804003

Polit, D., \& Tatano, C. (2006). The content validity index: Are you sure you know what's being reported? critique and recommendations. Research in Nursing \& Health, 29(5), 489-497. https://doi.org/10.1002/nur.20147

Rosales, F. (2019). Revisión literaria del rango apropiado de la presión del cuff para el manejo de usuarios adultos con vía aérea artificial. Revista de Investigación en Logopedia, 9(1), 51-66. https://doi.org/10.5209/RLOG.61482

Ross, K. (2005). Quantitative research methods in educational planning. Recuperado de http://www2.tf.jcu.cz/ bauman/KPD_VTMP_KVTMP/Educ ational_Research.pdf

Russell, C., \& Matta, B. (2004). Tracheostomy: A MultiProfessional Handbook. New York: Cambridge University Press.

Saleh, A., \& Bista, K. (2017). Examining Factors Impacting Online Survey Response Rates in Educational Research: Perceptions of Graduate Students. Journal of MultiDisciplinary Evaluation, 13(29), 63-74. Recuperado de http://journals.sfu.ca/jmde/index.php/jmde_1/article/vie $\mathrm{w} / 487$

Singh, A. S., Vik, F. N., Chinapaw, M. J. M., Uijtdewilligen, L., Verloigne, M., Fernández-Alvira, J. M., ... Brug, J. (2011). Test-retest reliability and construct validity of the ENERGYchild questionnaire on energy balance-related behaviours and their potential determinants: The ENERGY-project. The International Journal of Behavioral Nutrition and Physical Activity, 8, 136. https://doi.org/10.1186/1479-5868-8-136

Sole, M. L., Su, X., Talbert, S., Penoyer, D. A., Kalita, S., Jimenez, E., ... Bennett, M. (2011). Evaluation of an intervention to maintain endotracheal tube cuff pressure within therapeutic range. American Journal of Critical Care: An Official Publication, American Association of Critical-Care Nurses, 20(2), 109-117; quiz 118. https://doi.org/10.4037/ajcc2011661

Tools4dev. (2014). How to pretest and pilot a survey questionnaire.

University of Southampton. (2018). Research Data Management Policy University Regulations. Recuperado 15 de abril de 2018, de http://www.calendar.soton.ac.uk/sectionIV/research-datamanagement.html 